\title{
Free Convection Heat Transfer in a Micropolar Fluid Confined Between a Long Vertical Wavy Wall and a Parallel Flat Wall
}

\author{
Seema Goyal ${ }^{1^{*}}$ \\ ${ }^{1}$ Department of Maths, Meerut College, Meerut, UP \\ *Corresponding author: seemagoyal19@gmail.com
}

Received: 30.11.2021; Revised: 19.12.2021; Accepted: 26.12.2021

(C) Society for Himalayan Action Research and Development

\begin{abstract}
The free convection heat transfer in a micropolar fluid confined between a long vertical wavy wall and a parallel flat wall has been studied. Analysis of fluid flow over a wavy wall is of interest because of its physical applications such as transpiration cooling of re-entry vehicles, rocket booster and film vaporization, in combustion chambers etc. The equations governing the flow and the heat transfer have been solved subject to the relevant boundary conditions by assuming that the solution consists of two parts viz. a mean part and a perturbed one. To obtain the perturbed part of the solution, use has been made of the long Wavy approximation. The sets of differential equations have been solved by Finite Element Method. The zeroth order, the first order and total solution of the problem have been evaluated numerically for several sets of values of the various parameters entering the problem and are depicted graphically.
\end{abstract}

Keywords: Micropolar Fluid, Free Convection, Vertical Wavy Wall, Source/Sink, Nusselt Number

\section{Introduction}

Eringen(1966) put forward the theory of micro fluids which has been further extended to micropolar fluids that exhibits certain microscopic effects arising from the local structure and micro motion of the fluid elements. The governing equation of flow of micropolar fluid involve classical velocity vector, a micro rotation vector and gyration parameter. The concept of initial spin, micro stress averages, body moments and stress moments arise due to micro motion of fluid elements which have no counterpart in classical fluid theories. Some of the practical examples confirming to micropolar fluid model are liquid crystals, animal blood and blood flows Ariman (1971) and certain polymeric fluids. Modern applications of heat transfer analysis in various industries viz. nuclear, electronics, chemical and aerospace have motivated many developments in this field. Studies of heat convection in micropolar fluids have been focused mainly on flat plates Agarwal (1988) or regular surface Balaram (1973). A few investigators have examined the effects of complex geometries on natural convection in micropolar fluids. The problem of free convective heat transfer in a viscous fluid flowing over a vertical wavy wall and a parallel flat wall has been tackled by Vajravelu and Sastry (1978). Transient analysis of natural convection along a vertical wavy surface has been analysed by Chiu and Chou (1994). Umavathi, et all (2010) gives study of mixed convective flow of immiscible viscous fluids confined between a long vertical wavy wall and a parallel flat wall. 
In view of several important applications of such flow problems this paper has been devoted to problem of free convective heat transfer in a micropolar fluid confined between a long vertical wavy wall and a parallel flat wall. To solve this complicated problem, linearization technique is used. The solution is made up of two parts, mean part corresponding to the fully developed mean flow and the other a small disturbance.

\section{Mathematical formulation}

Consider the two-dimensional steady flow of an incompressible micropolar fluid confined to the vertical Wavy channel as shown in fig.1. In the channel $\mathrm{x}$ excess is taken vertically upwards and parallel to the flat wall while the $y$ axis is taken perpendicular to it. The wavy wall and the flat wall are represented by $Y=\epsilon^{*} \cos K X$ and $Y=d$ respectively and are maintained at constant temperatures $T_{w}$ and $T_{1}$

Introducing the non-dimensional variables

$$
x=\frac{X}{d}, \quad y=\frac{Y}{d}, \quad u=\frac{U d \rho}{\mu}, \quad v=\frac{V d \rho}{\mu} p=\frac{p^{*} d^{2} \rho^{2}}{\rho \mu^{2}}, \quad \lambda=K d, \quad \epsilon=\frac{\epsilon^{*}}{d}, \quad g=\frac{v d^{2} \rho}{\mu}, \quad \theta=\frac{T-T_{s}}{T_{W}-T_{s}}
$$

where $T_{s}$ is the fluid temperature in hydro static condition, the equation governing the flow and heat transfer problem are written as :

$$
\begin{aligned}
& u \frac{\partial u}{\partial x}+v \frac{\partial u}{\partial y}=G \theta+(1+R)\left[\frac{\partial^{2} u}{\partial x^{2}}+\frac{\partial^{2} u}{\partial y^{2}}\right]+R \frac{\partial g}{\partial y}-\frac{\partial\left(p-p_{s}\right)}{\partial x} \\
& u \frac{\partial v}{\partial x}+v \frac{\partial v}{\partial y}=-\frac{\partial p}{\partial y}+(1+R)\left[\frac{\partial^{2} v}{\partial x^{2}}+\frac{\partial^{2} v}{\partial y^{2}}\right]-R \frac{\partial g}{\partial x} \\
& u \frac{\partial g}{\partial x}+v \frac{\partial g}{\partial y}=A\left[\frac{\partial^{2} g}{\partial x^{2}}+\frac{\partial^{2} g}{\partial y^{2}}\right]+B\left[\frac{\partial v}{\partial x}-\frac{\partial u}{\partial y}-2 g\right] \\
& \operatorname{Pr}\left[u \frac{\partial \theta}{\partial x}+v \frac{\partial \theta}{\partial y}\right]=\frac{\partial^{2} \theta}{\partial x^{2}}+\frac{\partial^{2} \theta}{\partial y^{2}}+\alpha^{*}
\end{aligned}
$$

Also, the boundary conditions may be written as:

$$
\begin{aligned}
& y=\epsilon \cos \lambda x ; \quad u=0 \quad v=0 \quad g=\frac{1}{2}\left[\frac{\partial v}{\partial x}-\frac{\partial u}{\partial y}\right] \quad \theta=1 \\
& y=1 ; \quad u=v=0 \quad g=-\frac{1}{2}\left[\frac{\partial v}{\partial x}-\frac{\partial u}{\partial y}\right] \quad \theta=m
\end{aligned}
$$

where $\quad A=\frac{\gamma}{\mu j}, \quad B=\frac{\sigma \mathrm{d}^{2}}{\mu j}, \quad m=\frac{T_{1}-T_{s}}{T_{w}-T_{s}}, \quad R=\frac{k}{\mu}$

$$
\operatorname{Pr}=\frac{\mu C_{p}}{k_{f}}, \quad G=\frac{\mathrm{d}^{3} g_{s} \beta_{1}\left(T_{w}-T_{s}\right) \rho}{\mu^{2}}, \quad \alpha^{*}=\frac{Q \mathrm{~d}^{2}}{k_{f}\left(T_{w}-T_{s}\right)}
$$

Here $\alpha^{*}$ is the non-dimensional heat source/sink parameter, $m$, the wall temperature ratio, $\epsilon$, the nondimensional amplitude parameter.

In equation (2), the following hydro static condition has been used. 


$$
0=\frac{\partial p_{s}}{\partial x}-\frac{\rho_{s} g_{s} \mathrm{~d}^{3} \rho}{\mu^{2}}
$$

Using the method of perturbation, different functions are characterized as

$$
\begin{array}{ll}
u(x, y)=u_{0}(y)+u_{1}(x, y), & v(x, y)=v_{1}(x, y) \\
g(x, y)=g_{0}(y)+g_{1}(x, y), & p(x, y)=p_{0}(y)+p_{1}(x, y) \\
\theta(x, y)=\theta_{0}(y)+\theta_{1}(x, y) &
\end{array}
$$

where the perturbations $u_{1}, v_{1}, g_{1}, p_{1}$ and $\theta_{1}$ are small as compared with the mean or the zeroth-order quantities. Substituting (7) in (2-5), the zeroth order equations become as

$$
\begin{aligned}
& (1+R) \frac{\mathrm{d}^{2} u_{0}}{\mathrm{~d} y^{2}}+G \theta_{0}+R \frac{\mathrm{d} g_{0}}{\mathrm{~d} y}=0 \\
& A \frac{\mathrm{d}^{2} g_{0}}{\mathrm{~d} y^{2}}-B \frac{\mathrm{d} u_{0}}{\mathrm{~d} y}-2 B g_{0}=0 \\
& \frac{d^{2} \theta_{0}}{d y^{2}}=-\alpha^{*}
\end{aligned}
$$

While the first order equations are as follows:

$$
\begin{gathered}
u_{0} \frac{\partial u_{1}}{\partial x}+v_{1} \frac{\partial u_{0}}{\partial y}=G \theta_{1}+(1+R)\left[\frac{\partial^{2} u_{1}}{\partial x^{2}}+\frac{\partial^{2} u_{1}}{\partial y^{2}}\right]-\frac{\partial p_{1}}{\partial x}+R \frac{\partial g_{1}}{\partial y} \\
u_{0} \frac{\partial v_{1}}{\partial x}=-\frac{\partial p_{1}}{\partial y}+(1+R)\left[\frac{\partial^{2} v_{1}}{\partial x^{2}}+\frac{\partial^{2} v_{1}}{\partial y^{2}}\right]-R \frac{\partial g_{1}}{\partial y} \\
u_{0} \frac{\partial g_{1}}{\partial x}+v_{1} \frac{\partial g_{0}}{\partial y}=A\left[\frac{\partial^{2} g_{1}}{\partial x^{2}}+\frac{\partial^{2} g_{1}}{\partial y^{2}}\right]+B\left[\frac{\partial v_{1}}{\partial x}-\frac{\partial u_{1}}{\partial y}-2 g_{1}\right] \\
\operatorname{Pr}\left[u_{0} \frac{\partial \theta_{1}}{\partial x}+v_{1} \frac{\partial \theta_{0}}{\partial y}\right] \\
=\frac{\partial^{2} \theta_{1}}{\partial x^{2}}+\frac{\partial^{2} \theta_{1}}{\partial y^{2}}
\end{gathered}
$$

The boundary conditions (6) yield

$$
\begin{array}{ll}
u_{0}=0 \quad g_{0}=-\frac{1}{2} \frac{\partial u_{0}}{\partial y} \quad \theta_{0}=1, \quad \text { at } y=0 \\
u_{0}=0 \quad g_{0}=\frac{1}{2} \frac{\partial u_{0}}{\partial y} \quad \theta_{0}=m, \quad \text { at } y=1 \\
u_{1}=-u_{0}^{\prime} \quad v_{1}=0 \quad g_{1}=-\frac{1}{2}\left[\frac{\partial u_{1}}{\partial y}+\frac{d^{2} u_{0}}{\partial y^{2}}\right]-g_{0}^{\prime} \quad \theta_{1}=-\theta_{0}^{\prime}, \quad \text { at } \quad y=0 \\
u_{1}=0 \quad v_{1}=0 \quad g_{1}=\frac{1}{2} \frac{\partial u_{1}}{\partial y} \quad \theta_{1}=0, \quad \text { at } \quad y=1
\end{array}
$$

where a prime denotes differentiation with respect to $y$. To solve first order equations a stream function $\bar{\psi}_{\text {is }}$ introduced such that

$$
u_{1}=-\frac{\partial \bar{\psi}}{\partial y}, \quad v_{1}=\frac{\partial \bar{\psi}}{\partial x}
$$


Further eliminating $p_{1}$ from (11) and (12) and defining

$$
\begin{aligned}
& \bar{\psi}(x, y)=\mathrm{e}^{\mathrm{i} \lambda x} \Psi(y), \quad g_{1}(x, y)=\mathrm{e}^{\mathrm{i} \lambda x} s(y) \\
& \theta_{1}(x, y)=\mathrm{e}^{\mathrm{i} \lambda x} t(y)
\end{aligned}
$$

The following ordinary differential equations are obtained

$$
\begin{aligned}
& (1+R)\left[\Psi^{i v}-2 \lambda^{2} \Psi^{\prime \prime}+\lambda^{4} \Psi\right]+\mathrm{i} \lambda\left[-u_{0} \psi^{\prime \prime}+\psi u_{0}^{\prime \prime}+u_{0} \lambda^{2} \psi\right]-R s^{\prime \prime}-R \lambda^{2} s=G t^{\prime \prime} \\
& A\left[s^{\prime \prime}-\lambda^{2} s\right]-\mathrm{i} \lambda\left[u_{0} s+\psi g_{0}^{\prime}\right]+B\left[-\lambda^{2} \Psi+\psi^{\prime \prime}-2 s\right]=0 \\
& t^{\prime \prime}-\lambda^{2} \Psi=i \lambda \operatorname{Pr}\left[u_{0} t+\theta_{0}^{\prime \prime} \Psi\right]
\end{aligned}
$$

subject to boundary conditions

$$
\begin{array}{lll}
\Psi^{\prime}=u_{0}^{\prime} & \Psi=0 & s=\frac{1}{2} \Psi^{\prime \prime}(0)-\frac{1}{2} u_{0}^{\prime \prime}-g_{0}^{\prime} \quad t=-\theta_{0}^{\prime}, \quad \text { at } y=0 \\
\Psi^{\prime}=0 \quad \Psi=0 \quad s=-\frac{1}{2} \Psi^{\prime \prime}(1) \quad t=0, \quad \text { at } \quad y=1
\end{array}
$$

Taking $\lambda$ to be small and substituting

$$
\psi=\sum_{j=0}^{2} \lambda^{j} \psi_{j}, \quad t=\sum_{j=0}^{2} \lambda^{j} t_{j}, \quad s=\sum_{j=0}^{2} \lambda^{j} s_{j}
$$

into (15-17), the following sets of equations are obtained

$$
\begin{aligned}
& (1+R) \Psi_{n}^{i v}-R s_{n}^{\prime \prime}-G t_{n}^{\prime}=0 \\
& A s_{n}^{\prime \prime}+B \Psi_{n}^{\prime \prime}-2 B s_{0}=0 \\
& t_{n}^{\prime \prime}=0 \\
& (1+R) \Psi_{1}^{i v}-R s_{1}^{\prime \prime}-G t_{1}^{\prime}+i\left(-u_{0} \Psi_{0}^{\prime \prime}+\Psi_{0}^{\prime \prime \prime}\right)=0 \\
& A s_{1}^{\prime \prime}-i\left(u_{0} s_{0}+\Psi_{0}^{\prime} g_{0}^{\prime}\right)+B\left(\Psi_{1}^{\prime \prime}-2 s_{1}\right)=0 \\
& t_{1}^{\prime \prime}=i \operatorname{Pr}\left(u_{0} t_{0}+\theta_{n}^{\prime} \Psi_{0}\right) \\
& (1+R) \Psi_{2}^{i v}-2(1+R) \Psi_{0}^{\prime \prime}-R s_{2}^{\prime \prime}+R s_{0}+i\left(-\Psi_{1}^{\prime \prime} u_{0}+\Psi_{1} u_{0}^{\prime \prime}\right)-G t_{2}^{\prime}=0 \\
& A s_{2}^{\prime \prime}-A s_{0}-i\left(u_{0} s_{1}+\Psi_{1}^{\prime} g_{0}^{\prime}\right)+B\left(-\Psi_{0}+\Psi_{2}^{\prime \prime}-2 s_{2}\right)=0 \\
& t_{2}^{\prime \prime}-t_{0}=i \operatorname{Pr}\left(u_{0} t_{1}+\theta_{0}^{\prime} \Psi_{1}\right)
\end{aligned}
$$

The boundary conditions (18) accordingly change as

$$
\begin{array}{llll}
\Psi_{0}=u_{0} & \Psi_{0}=0 & s_{0}=-g_{0}^{\prime}-\frac{1}{2} u_{0}^{\prime \prime}+\frac{1}{2} \Psi_{0}^{\prime \prime} \quad t_{0}=-\theta_{0}^{\prime}, \quad \text { at } y=0 \\
\Psi_{0}^{\prime}=0 & \Psi_{0}=0 & t_{0}=0 \quad s_{1}=\frac{1}{2} \Psi_{1}^{\prime \prime}, \quad \text { at } y=1 \\
\Psi_{i}^{\prime}=0 & \Psi_{i}=0 \quad s_{i}=\frac{1}{2} \Psi_{i}^{\prime \prime} \quad t_{i}=0, \quad \text { at } y=0 \\
\Psi_{i}^{\prime}=0 & \Psi_{i}=0 \quad s_{i}=-\frac{1}{2} \Psi_{i}^{\prime \prime} \quad t_{i}=0, \quad \text { at } y=1 \text { for } i \geq 1
\end{array}
$$


The shear stress at any point in the fluid is given by

$$
\tau_{x y}=\mu\left[\frac{\partial U}{\partial x}+\frac{\partial V}{\partial y}\right]+k\left[\frac{\partial V}{\partial y}+v\right]
$$

In dimensionless form, the shear stress is

$$
\tau_{x y}=\frac{d^{2} \tau_{x y}}{\rho v^{2}}=\left[\mu+\frac{K}{2}\right]\left[\frac{\partial u}{\partial x}+\frac{\partial v}{\partial y}\right]
$$

At the wavy wall $y=\epsilon \cos \lambda x$ and at the flat wall $y=1$, the shear stresses become as

$\tau_{w}=\tau_{0}^{0}+\frac{(2+R)}{2} \operatorname{Re}\left[\epsilon \mathrm{e}^{\mathrm{i} \lambda x} u_{0}^{\prime \prime}(0)+\epsilon \mathrm{e}^{\mathrm{i} \lambda x} u_{1}^{\prime}(0)\right]$

and $\quad \tau_{1}=\tau_{1}^{0}+\frac{(2+R)}{2} \operatorname{Re}\left[\epsilon \mathrm{e}^{\mathrm{i} \lambda x} u_{1}^{\prime}(1)\right]$

where $\tau_{0}^{0}=\frac{(2+R)}{2} u_{0}^{\prime}(0)$ and $\tau_{1}^{0}=\frac{(2+R)}{2} u_{0}^{\prime}(1)$

The wall couple stress coefficient is given by $C_{m}=\gamma \frac{\partial v}{\partial y}$ which in dimensionless form becomes as

$$
M=\frac{\rho C_{m}}{\mu^{2} j d}=A \frac{\partial g}{\partial y}
$$

Accordingly on the walls, they are written as

$$
M_{\mathrm{w}}=M_{\mathrm{n}}^{0}+\operatorname{ARe}\left[\epsilon \mathrm{e}^{\mathrm{i} \lambda x} q_{\mathrm{n}}^{\prime \prime}(0)+\epsilon \mathrm{e}^{\mathrm{i} \lambda x} g_{1}^{\prime}(0)\right\rceil
$$

and $\quad M_{1}=M_{1}^{0}+$ Are $\left\lceil\epsilon \mathrm{e}^{\mathrm{i} \lambda x} g_{1}^{\prime}(1)\right\rceil$

where $M_{\mathrm{n}}^{0}=A g_{\mathrm{n}}^{\prime}(0), \quad M_{1}^{0}=A g_{\mathrm{n}}^{\prime}(1)$

In a similar way the heat transfer coefficient $q_{w}$ is defined as

$$
q_{w}=-K_{f} \frac{\partial T}{\partial y}
$$

Which in non-dimensional form becomes

$$
\begin{aligned}
q_{w}=- & K_{f} \frac{\left(T_{w}-T_{s}\right)}{d}\left[\frac{d \theta_{0}}{d y}\right. \\
& \left.+\operatorname{Re}\left(\epsilon \mathrm{e}^{\mathrm{i} \lambda x} \frac{d \theta_{1}}{d y}\right)\right] \\
N_{u}= & \frac{d q_{w}}{T_{w}-T_{s}}=\theta_{0}^{\prime}(y)+\operatorname{Re}\left(\epsilon \mathrm{e}^{\mathrm{i} \lambda x} \theta_{1}^{\prime}(y)\right)
\end{aligned}
$$

Then, the Nusselt numbers on the walls are given by

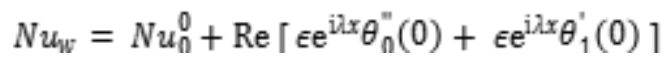

$$
\begin{aligned}
& N u_{1}=N u_{1}^{0}+\operatorname{Re}\left\lceil\epsilon \mathrm{e}^{\mathrm{i} \lambda x} \theta_{1}^{\prime \prime}(1)\right\rceil
\end{aligned}
$$

respectively where $N u_{n}^{0}=\theta_{n}^{\prime}(0), \quad N u_{1}^{0}=\theta_{n}^{\prime}(1)$ 
Method of solution- The four sets of coupled differential equations (8-10), (19), (20), (21) subject to the boundary conditions (15) and (23) have been sold separately by finite element method. The first order quantities $u_{1}, v_{1}, g_{1}$ and $\theta_{1}$ are then calculated as

$$
\begin{aligned}
& u_{1}=-\epsilon\left[\cos (\lambda x) \Psi_{r}^{\prime}-\sin (\lambda x) \Psi_{i}^{\prime}\right] \\
& v_{1}=-\epsilon \lambda\left[\sin (\lambda x) \Psi_{r}+\cos (\lambda x) \Psi_{i}\right] \\
& g_{1}=\epsilon\left[\cos (\lambda x) s_{r}-\cos (\lambda x) s_{i}\right] \\
& \theta_{1}=\epsilon\left[\cos (\lambda x) t_{r}-\cos (\lambda x) t_{i}\right]
\end{aligned}
$$

Where $\Psi=\Psi_{r}+i \Psi_{i}, \quad \Psi^{\prime}=\Psi_{r}^{\prime}+i \Psi_{i}^{\prime}$

$$
s=s_{r}+i s_{i}, \quad t=t_{r}+i t_{i}
$$

The expressions for $u_{1}, v_{1}, g_{1}$ and $\theta_{1}$ are the disturbed part of the corresponding functions due to waviness of the wall.

\section{Results and Discussion}

Zeroth order Solution- The behaviour of the non-dimensional zeroth order velocity $u_{0}$ of the fluid with variation in micropolar parameter $R$, the free Convection parameter $G$, and the heat source parameter $\alpha^{*}$ is depicted in fig. 2 for the case $m=-1$ (Physically, $m=-1$ means that the average of the temperatures of the two walls is equal to that of the static fluid) and in fig. 3 for $m=2$ (wall temperature unequal). From fig. 2 , it is clear that, with an increase in micropolar parameter $R$, the magnitude of fluid velocity $u_{0}$ decreases across the entire channel width for all $\alpha^{*}$ but the reverse happens with $G$. In the presence of heat sources $\left(\alpha^{*}>0\right.$, curves V, VI, VII) the fluid velocity decreases across the channel width with the increase in micropolar parameter $R^{\prime}$ while increases whenever the free convection parameter $G$ increases. This behaviour is reversed in the case of heat sinks $\alpha^{*}<0$, curves I, II, III, IX, X). On fixing $R$ and
$G$ and varying $\alpha^{*}$, we observe that with an increase in the heat source parameter $\alpha^{*}$, the fluid velocity increases considerably.

From fig. 3, it is obvious that for $m=2$ the fluid velocity $u_{0}$ is enhanced by an increase in the free convection parameter $G$ for all values of $\alpha^{*}$ but decreases with an increase in $R$. Close examination of fig. 2 and fig. 3 reveals that the fluid velocity can reverse its direction in the case $m=-1$ while there is no such possibility when $m \geq 0$ which is physically true because for $m \geq 0$, the temperature of one wall exceeds than that in the hydrostatic state while on the other wall, lies below that. Fig. 4 and fig.5 depict the micro rotation function for $m=-1$ and $m=2$ respectively.

The behaviour of the fluid temperature with changes in $\alpha^{*}$ is shown for $m=-1$ in fig. 6 and for $m=2$ in fig.7. From fig. 6, it is clear that in the absence of heat sources $\left(\alpha^{*}=0\right)$ the 
fluid temperature $\theta_{0}$ is a linear function of $y$, while in the presence of heat sources/sinks $\left(\alpha^{*}>0 / \alpha^{*}<0\right)$ the temperature is parabolic in nature. Also, it decreases from its value at the wall $y=0$ to a minimum temperature near $y=0.9$ and then increases steadily to its value at $y=1$ for $\alpha^{*}<0$ while the profile for $\alpha^{*}>0$ is exactly the opposite. Fig. 8 shows that the zeroth order skin friction at either wall is a linear function of the heat source parameter $\alpha^{*}$ and that the skin friction at the wall $y=0$ increases with the increase in $\alpha^{*}$ while the reverse is true at the other wall $y=1$. The skin friction $m=-1$ at $y=0$ is a decreasing function of $R$ and increasing function of $G$ while that at $y=1$ the magnitude of skin friction has the same pattern. For Fig. 9 gives the zeroth order couple stress coefficients whose variation resembles to that of the skin friction.

The heat transfer coefficient $N u^{0}$ increases with increasing $\alpha^{*}$ at $y=0$ and decreases at the other wall for all values of $m$ ( fig. 10). Also, the rate of heat transfer at either wall increases with an increase in $m$.

First order solution - Fig. 11-14 depict the behaviour of perturbed quantities $u_{1}, v_{1}, g_{1}$ and $\theta_{1}$ when $m=-1$ and Prandtl number
$\operatorname{Pr}=0.71$. From fig. 11 , it is observed that in the presence of heat sinks the fluid velocity $u_{1}$ increases steadily upto $y=0.5$ i.e. in the first half of the channel, while in the other half $u_{1}$ decrease with $y$. In the presence of heat sources as well as no heat sources, the pattern of fluid velocity $u_{1}$ becomes reverse to the previous case. Also the magnitude of perturb velocity $u_{1}$ increases with a decrease in micropolar parameter $R$ and an increase in free convection parameter $G$ in the presence of heat sources (curves V, VI, VII) and also for heat sinks (curves I, II, IX, X). Fig. 12 shows that the behaviour of fluid velocity $v_{1}$ perpendicular to the channel length for $\lambda x=\pi / 2$. Fig. 13 describes the behaviour of micro rotation $g_{1}$ for $\lambda x=0$. It is clear from the figure that in the presence of heat sinks, the micro rotation remains positive while for heat sources and no heat sources, the micro rotation is negative throughout the channel. Fig 14 analyses the behaviour of fluid temperature $\theta_{1}$ for $\lambda x=0$. The first order temperature profile is an increasing function of $\alpha^{*}>0$ while for $\alpha^{*}<0$ the behaviour is reversed. 


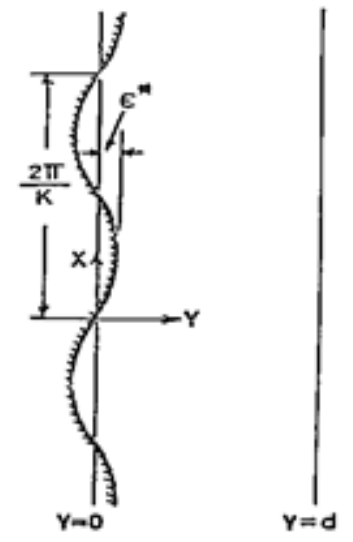

Fig. 1 Flow Configuration

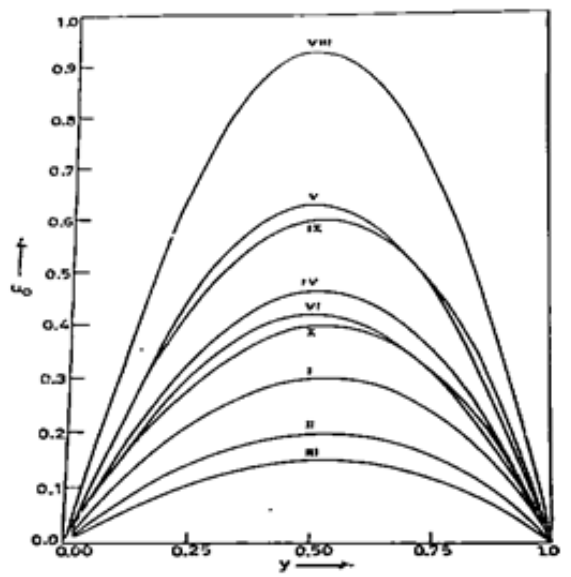

Fig. 3 Dimensionless zeroth-order velocity profiles $m=2$

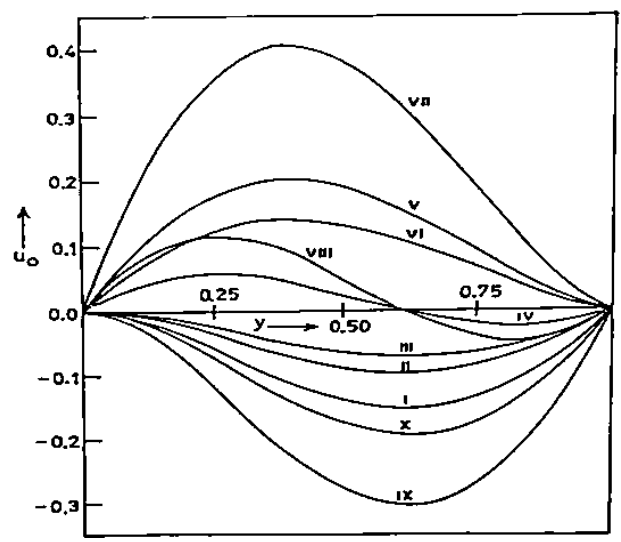

Fig. 2 Dimensionless zeroth-order velocity profiles $m=-1$

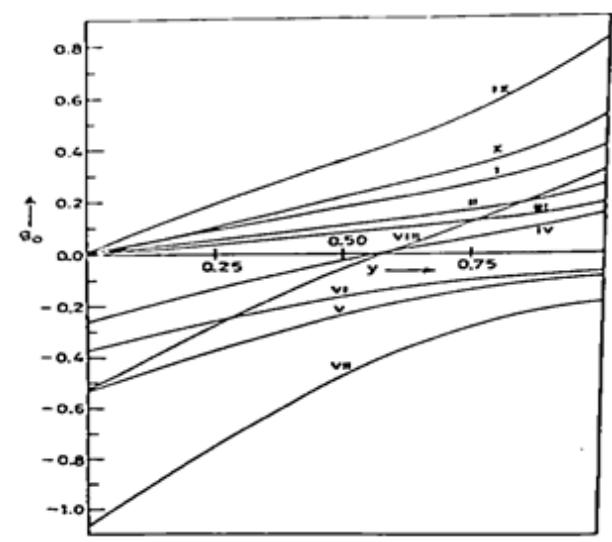

Fig. 4 Dimensionless zeroth-order microrotation profiles $m=-1$

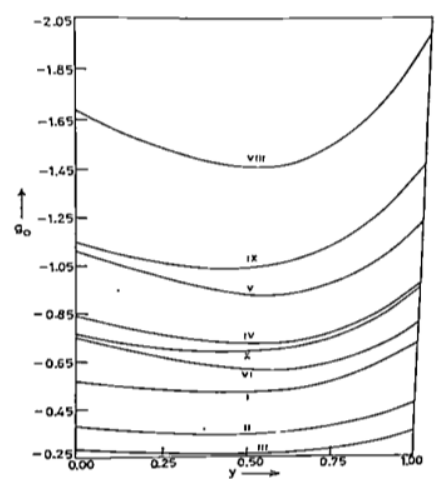

Fig. 5 Dimensionless zeroth-order microrotation profiles $m=2$

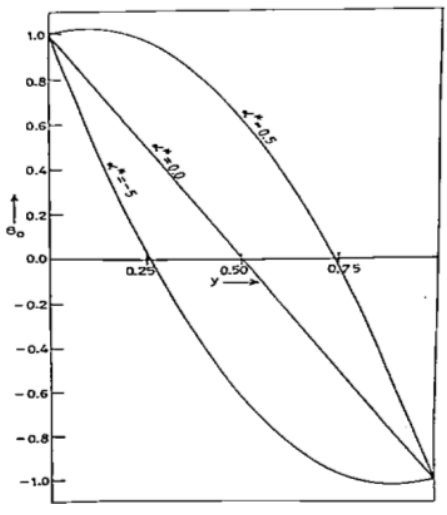

Fig. 6 Dimensionless zeroth-order temperature profiles $m=-1$ 


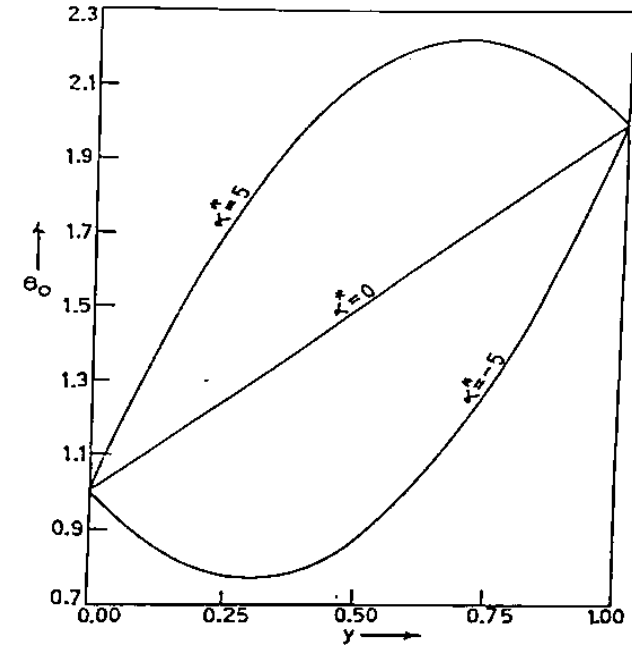

Fig. 7 Dimensionless zeroth-order temperature profiles $m=2$

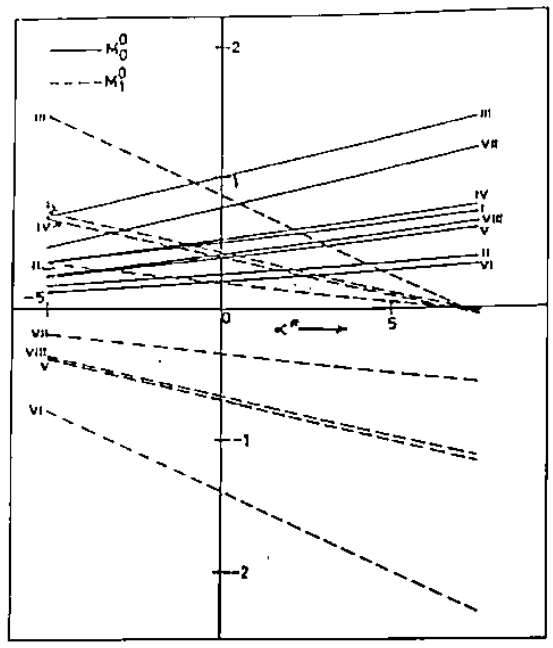

Fig. 9 Zeroth order couple stress at $y=0$ and $y=1$

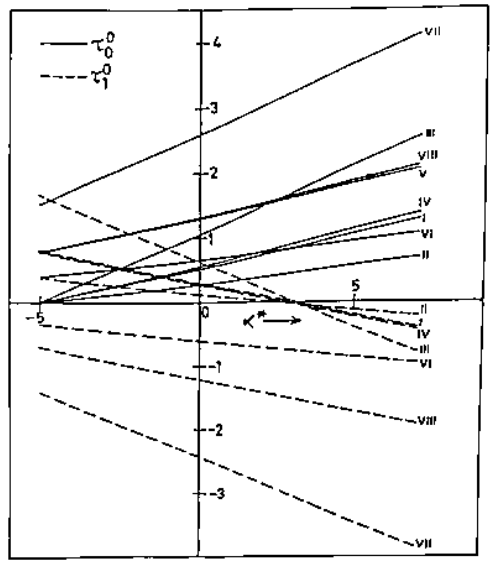

Fig. 8 Zeroth order skin friction at $y=0$ and $y=1$

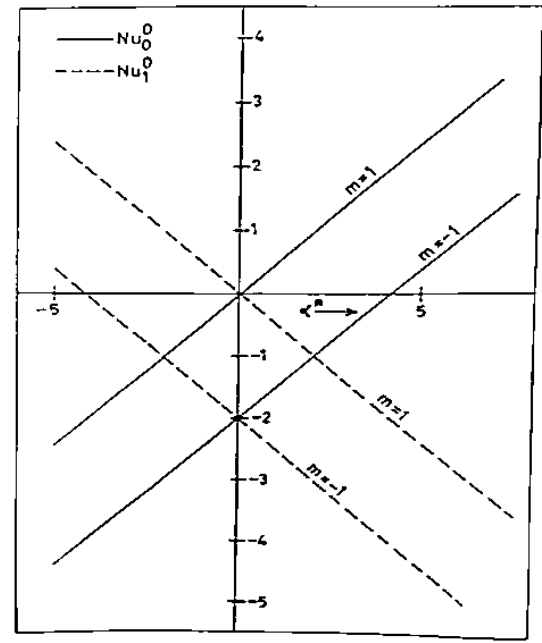

Fig. 10 Zeroth order Nusselt number at $y=0$ and $y=1$ 


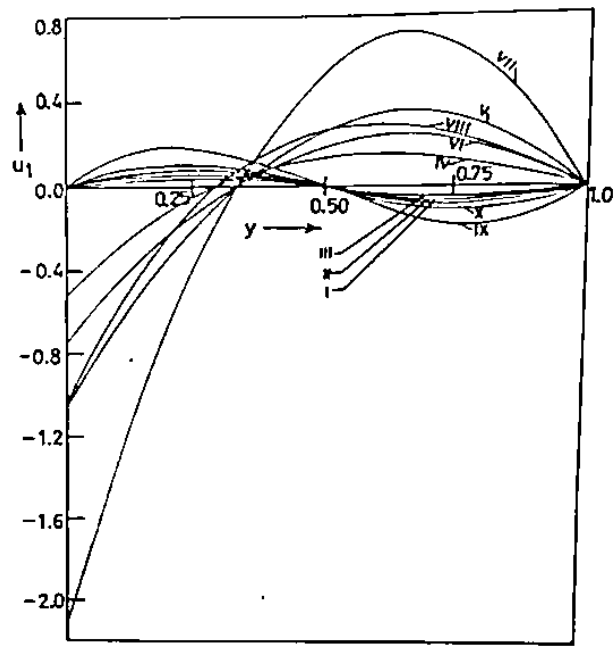

Fig. 11 Dimensionless first order velocity profile $u_{1}, m=-1, \operatorname{Pr}=0.71, \lambda=0.01, \lambda x=0$

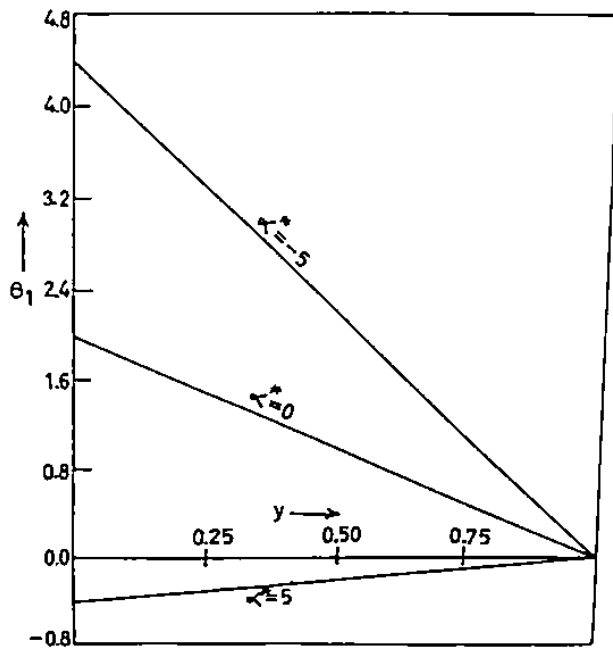

Fig. 13 Dimensionless first order microrotation profile $u_{1}, m=-1, \operatorname{Pr}=0.71, \lambda=0.01, \lambda x=0$

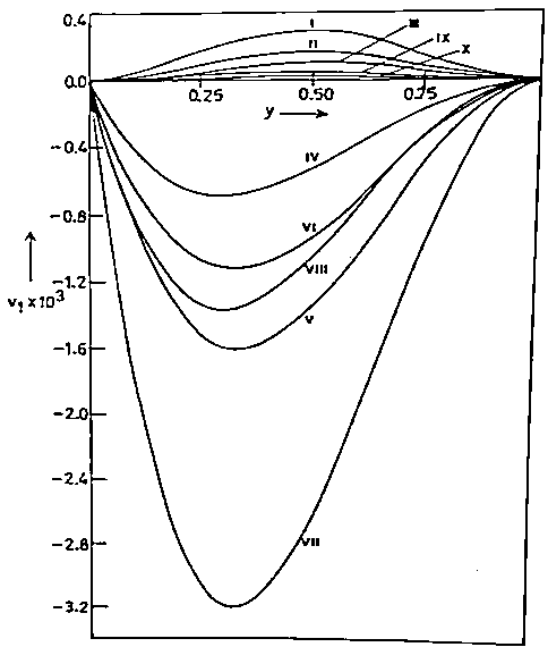

Fig. 12 Dimensionless first order velocity profile $v_{1}, m=-1, \operatorname{Pr}=0.71, \lambda=0.01, \lambda x=\pi / 2$

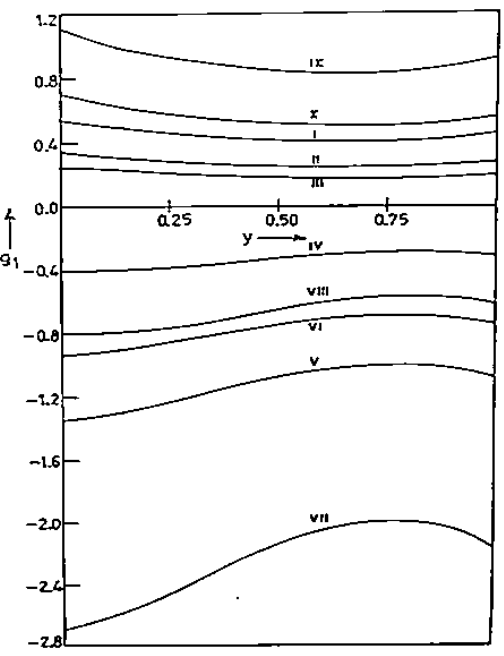

Fig. 14 Dimensionless first order temperature profile $u_{1}, m=-1, \operatorname{Pr}=0.71, \lambda=0.01, \lambda x=0$

For Figure 2-5 and 11-13, the curves (I-X) are

\begin{tabular}{|c|c|c|c|c|c|c|c|c|c|c|c|c|c|c|c|c|c|c|c|}
\hline \multirow{2}{*}{\multicolumn{2}{|c|}{ I }} & I & II & I & $V$ & $\mathrm{~V}$ & VI & VII & I & $\mathbf{Y}$ & \multicolumn{8}{|c|}{ For figure $8-9$, the curves (I-VIII) are } & \multirow[b]{2}{*}{ VIII } \\
\hline & & I & I & $\mathrm{V}$ & & I & I & I & $\mathrm{X}$ & $\Lambda$ & & I & II & III & IV & V & VI & VII & \\
\hline$R$ & 1 & 2 & 3 & 1 & 1 & 2 & 1 & 1 & 1 & 2 & $R$ & 1 & 3 & 1 & 3 & 1 & 3 & 1 & 3 \\
\hline \multirow[t]{2}{*}{$G$} & \multirow[t]{2}{*}{5} & \multirow[t]{2}{*}{5} & \multirow[t]{2}{*}{5} & \multirow[t]{2}{*}{5} & \multirow[t]{2}{*}{5} & \multirow[t]{2}{*}{5} & \multirow[t]{2}{*}{10} & \multirow[t]{2}{*}{10} & \multirow[t]{2}{*}{10} & 1 & $G$ & 5 & 5 & 10 & 10 & 5 & 5 & 10 & 10 \\
\hline & & & & & & & & & & 0 & $m$ & -1 & -1 & -1 & -1 & 1 & 1 & 1 & 1 \\
\hline & 5 & 5 & -5 & 0 & 5 & 5 & 5 & 0 & -5 & -5 & & & & & & & & & \\
\hline
\end{tabular}




\section{References}

Agarwal, R.S. and Dhanpal, C. (1988) Numerical solution of free convection fluid flow between two parallel porous vertical plate, Int. J. Eng.Sci., 260, 1247.

Ariman, T. (1971) On the analysis of blood flow, J. Biomech., 4, 185.

Balaram, M. and Sastri, V. U. K. (1973) Micropolar free convection flow, Int. J. of Heat and Mass Transfer, 16, 437.

Chiu, Cheng-Ping and Chou, Huann- Ming (1994) Transient analysis of natural convection along a vertical wavy surface in micropolar fluids, Int. J. Eng. Sci., 32, 19.

Eringen, A. C. (1966) Theory of micropolar fluids, J. Math. Mech., 16,1.

Hung, K. Y., Hsu, T. H., Lin, J. W. (2013) Mixed convection of micropolar fluids in a vertical wavy channel saturated with porous media, 16, 1107.

Umavathi, J. C., Prathap,J.,Shekar, M. (2010) Mixed convective flow of immiscible viscous fluids confined between a long vertical wavy wall and a parallel flat wall, Int. J. of Eng. Sci. and Tech., 2, 256.

Vajravelu, K. and Sastry, K. S. (1978) Free convective heat transfer in a viscous incompressible fluid confined between a long vertical wavy wall and parallel flat wall, J. Fluid Mech., 86, 365. 